\section{Findings from 2017 on Health Information Management}

\author{
Meryl Bloomrosen', Eta S. Berner'2, Section Editors for the IMIA Yearbook Section on Health \\ Information Management \\ 1 Premier healthcare alliance, Washington, DC, USA \\ 2 Graduate Programs in Health Informatics, Department of Health Services Administration, \\ University of Alabama at Birmingham, Birmingham, AL, USA
}

\section{Introduction}

The issues of patient privacy and access to health information have always been central to the fields of Health Information Management (HIM) and Health Informatics. Health Information Managers often assume roles such as Chief Privacy Officer or other positions in healthcare organizations where they are advocates for patient privacy. Health and medical informatics professionals, on the other hand, have often focused on fostering broader access to health information for research and other secondary uses [1, 2], as well as on developing methods and models for health information exchange for clinical use [3]. At the same time, many informatics efforts have gone into developing new technological approaches to privacy and security protections when information is exchanged or shared $[4,5]$. Recent policy trends and changes in health information technology have made the inherent tension between these two goals more intense in recent years [6]. For instance, in the U.S., federal efforts to promote health information exchange, beginning with the Health Information Technology for Economic and Clinical Health (HITECH) Act [7] and continuing with the $21^{\text {st }}$ Century Cures Act have put pressure on electronic health records (EHRs) developers to make them improve the interoperability of their systems, and have incentivized healthcare organizations to implement health information technology (IT) and to engage in health information exchange [7, 8]. Changes in the Common Law promoted by the Office of Human Research Protection have made the secondary use of health information easier as well [9]. At the same time, the Health
Insurance Portability and Accountability Act (HIPAA) regulations have gotten more stringent in terms of accountability and penalties for privacy violations [10]. The articles that are included in this synopsis reflect the recent developments to address the challenges posed by public policy changes as well as the emergence and implementation of new health information technologies, functions, and capabilities.

\section{Methods}

At the end of December 2017, with the assistance of a medical librarian, the editors of the HIM section of the International Medical Informatics Association (IMIA) Yearbook conducted a search of HIM-related articles in both PubMed and Embase, using both MeSH descriptors and keywords in the titles and abstracts. The publication year was 2017 and did not include those articles that were e-published ahead of print. The query for PubMed was: "Health Information Management" [MSH] OR "Health Information Management" [tiab] OR "HIM J"[Journal] OR "JAHIMA"[Journal]", which yielded 198 results. The query for EMBASE was 'medical information system'/exp/mj OR "health information management":ti,ab OR "clinical information system":ti,ab OR "clinical pharmacy information systems":ti,ab OR "health information exchange":ti,ab OR "health information management":ti,ab OR "health information manager":ti,ab OR "health information network":ti,ab OR "health information system":ti,ab OR "health information systems":ti,ab OR "IS-H med":ti,ab OR "medical information service":ti,ab OR 
'Health Information Management Journal', which, yielded 382 articles. From these articles we excluded those with no title and/ or no abstract, which eliminated 63 articles, and finally we removed 100 duplicates from the remainder leaving 417 unique articles in English to review.

The 417 unique articles were rated by both section editors, who excluded articles that were opinion pieces or editorials, or articles where the full text of the article was not readily available. Each of the two section editors judged independently the relevance to the HIM field and the quality of the retrieved articles. Those that both co-editors rated as not appropriate were excluded automatically. The rest of the articles were discussed and disagreements adjudicated to arrive at 15 candidate best articles that, based primarily on the abstracts, were judged to be of good quality and to reflect the diverse aspects of the theme of the 2018 edition of the Yearbook. The full texts of these 15 articles were then rated independently by both section editors, one of the Yearbook editors, and at least two external peer reviewers. One of the articles, in hindsight, was not relevant to the theme of this year's Yearbook and is not discussed in this synopsis.

The four HIM-related 'Best Papers' were selected based primarily on having a high average rating from the reviewers. Other factors included the consensus of reviewers for including the paper as one of the best papers, as well as diversity of research approaches and settings.

The survey paper for the 2018 HIM section [11] presents a complementary focus to the research articles in that the survey paper focuses primarily on public policy issues related to privacy, access, and disclosure of health information. The authors, Kloss, Brodnik, and Rinehart-Thompson, performed a scoping literature review that examined regulations, policies, and laws related to access and disclosure of information, as well as practices designed to protect privacy and address problems with information privacy. Their review covers policies across multiple countries, and includes comparisons across country laws and practices. They noted key information and data governance issues that must be addressed and identified areas that are still in need of research, es-

Table 1 Best paper selection of articles for the IMIA Yearbook of Medical Informatics 2018 in the section 'Health Information Management'. The articles are listed in alphabetical order of the first author's surname.

\section{Section}

Health Information Management

- Boockvar KS, Ho W, Pruskowski J, DiPalo KE, Wong JJ, Patel J, Nebeker JR, Kaushal R, Hung W. Effect of health information exchange on recognition of medication discrepancies is interrupted when data charges are introduced: results of a cluster-randomized controlled trial. J Am Med Inform Assoc 2017 Nov 1;24(6):1095-101.

- Downing NL, Adler-Milstein J, Palma JP, Lane S, Eisenberg M, Sharp C; Northern California HIE Collaborative, Longhurst CA. Health information exchange policies of 11 diverse health systems and the associated impact on volume of exchange. J Am Med Inform Assoc 2017 Jan;24(1):113-22.

- Klein DM, Pham K, Samy L, Bluth A, Nazi KM, Witry M, Klutts JS, Grant KM, Gundlapalli AV, Kochersberger G, Pfeiffer L, Romero S, Vetter B, Turvey CL. The Veteran-Initiated Electronic Care Coordination: A Multisite Initiative to Promote and Evaluate Consumer-Mediated Health Information Exchange. Telemed J E Health 2017 Apr;23(4):264-27.

- Roehrs A, da Costa CA, da Rosa Righi R. OmniPHR: A distributed architecture model to integrate personal health records. J Biomed Inform 2017 Jul; 71:70-81.

pecially with the development of new technologies, new approaches and mechanisms of access to information, as well as current and contemplated legislation. Below we discuss the major themes illustrated by the 15 candidate best papers.

\section{Results}

\section{Health Information Exchange Policies and Governance}

Several articles addressed policy and governance issues in health information exchange (HIE) that must be considered for HIE to be successful and viable over the long-term in providing access to health information [1214]. The articles represented examples of health information exchange conducted in three different countries (Korea, Switzerland, and the U.S.) but many of the issues are common to all. There are legal and regulatory issues that must be dealt with related to information access and sharing, and policies surrounding standards, both in terms of data elements and interoperability [12]. The article by De Pietro and Francetic discusses changes in the laws that helped to promote the use of EHRs and health information exchange in Switzerland[13]. They also note that the Swiss health system as a whole is very fragmented which adds to the difficulties of health information exchange. This fragmentation is not unique to
Switzerland, although the ways in which it is displayed may be, and policies to address such fragmentation are needed in many countries. One of the key policy issues to address surrounds patient consent. The study by Downing et al., one of the 2018 best papers, discussed in more detail below, collected data on consent policies across 11 health systems and examined the relationship between the method for obtaining consent and the actual volume of information exchanged [14]. The researchers found that incorporating consent for HIE as part of the consent for treatment process, rather than requiring a separate consent each time health exchange was desired, led to an increased volume of information exchange. Similarly, they found that automating the process of obtaining records from another institution led to a similar outcome of more data exchanged. Given the importance of developing policies around consent for HIE, Downing et al.'s conclusions can be very useful in establishing reasonable policies that incorporate appropriate privacy protections, yet do not create unnecessary barriers to exchange.

\section{Architectures for Information Integration and Health Information Exchange}

Several of the 15 candidate best papers conceptualized and/or developed different architectures for integrating and exchanging health information. There was a focus on 
methods for integrating and making patient's personal health records (PHRs) accessible to their health care providers. The field of health information management has long had an interest in PHRs [15] and with the growing interest in patient-reported outcomes and their integration into EHRs [16], innovative strategies are needed to address incorporating PHR data into routine clinical care. One approach is to integrate information in the cloud. Bell et al., developed a conceptual model based on cloud-based platforms for making immunization data from multiple sources accessible to patients and providers [17]. Miyaji et al., used the multiparty private set interaction protocol as a method of integration of data [18]. Abdulnabi et al., suggested using smartphones to provide a distributed method of health information exchange, since most patients have access to smartphones [19]. Roehrs et al., another of the 2018 best papers, discussed in more detail below, developed and evaluated what the authors refer to as OminiPHR, which is also a distributed model, but uses blockchain technology [20]. Blockchain, while not currently used extensively in healthcare, has received considerable attention for potential healthcare applications and the article by Roehrs et al., suggests using it to make personal health record data accessible to patients and providers [21]. Collectively, these studies illustrate the challenges in creating efficient and workable solutions to integrating PHR and EHR data.

\section{Authentication and De-Identification}

In addition to developing methods for integrating data in the context of health information exchange, privacy and security issues must be addressed. Assuring privacy and security of healthcare data, whether access is through health information exchange or other means, is a focus of several articles. Park and Park [22] and Mohit et al. [23] developed innovative authentication approaches to address the major security issue of assuring that only those with the right to access information can obtain it. Aslam et al., reviewed a variety of authentication schemes, developed a new metric to evaluate these approaches, and suggested that patients and physicians might need different levels of authentication. They suggested two-factor authentication for patients, but three-factor approaches for health care providers [24].

In addition to authentication issues, some of the privacy issues involve de-identification. This may be particularly important with sensitive information such as substance abuse or mental illness which often have more stringent privacy regulations. Using Natural Language Processing techniques, Lee et al., developed and tested a novel approach to the de-identification of psychiatric notes [25]. As concerns about cybersecurity increase and as more and more electronic health information is generated, shared, and exchanged, the development, implementation, and evaluation of new approaches to authentication and de-identification will become even more important.

\section{Implementation of Health Information Exchange}

In addition to the foundational research needed to develop viable methods of HIE, research is also needed on strategies for implementation. The final set of papers from this year's selection illustrates a variety of approaches, settings, and technologies for health information exchange. One of the studies, by Klein et al., discussed in more detail in the best paper summaries, was done across nine hospitals within the US department of Veterans' Affairs (VA) [26]. Within the VA system, patients' information is accessible to all clinicians who need it, but there is an increasing need for exchange of data with private physicians outside the VA. The method of exchange decided upon was 'consumer-mediated' in that patients were taught how to download their VA health information so they could make it accessible to their outside providers. Klein et al., also evaluated the extent to which VA information was valuable to outside (non-VA) providers. The researchers found that most of the patients printed out the information for their outside providers, rather than exchanging it electronically. They also found that the outside providers appreciated having access to the data. This study illustrates that there are still challenges to the electronic exchange of information, but it is encouraging that if the information is easily accessible, even if not electronically, it is appreciated and used.

The last two papers focused on the importance of medication information. One of them, by Boockvar et al., also included as one of the best papers, was a cluster-randomized controlled trial examining the impact of the availability of pharmacy insurance claims data on medication discrepancies discovered during the medication reconciliation process for hospitalized patients [27]. They found that this data improved the medication reconciliation process in that when pharmacy claims data were available, there were more discrepancies discovered between pre-admission and inpatient medications. The discovery of these discrepancies and their reconciliation is important to improve patient safety. The final study by Hohmeier et al., looked at the reverse aspect of medication reconciliation [28]. These researchers examined how data on the patient's hospital course could impact what the community pharmacist does. This study was a qualitative study which examined how pharmacists utilize the data from a health information exchange. The authors found that it was feasible to integrate the data from HIE into the workflow of community pharmacists, and that pharmacists found that doing so aided identification of medication discrepancies. However, they also found that there was often a lag between patient discharge from the hospital and information availability from HIE. These three studies illustrate that health information exchange is becoming more feasible, that the data are perceived as useful, and that use of the data can improve patient safety. However, they also show that there are still limitations that must be addressed if the potential of HIE is going to be realized.

\section{Conclusion}

The set of papers in this year's HIM section of the Yearbook provide insights on the challenges of making health information easily accessible to providers and patients, keeping it secure, assuring privacy, fitting the exchange of information into the workflows 
of all who need it, and making optimal use of the data. Some of the papers addressed the developments of new technologies for data integration, authentication, and de-identification, while others focused on policy issues, implementation issues, and outcomes of the use of the data. This set of papers is a start, but more research on all of these areas policies, technologies, implementation, and outcomes - is needed for health information exchange to realize its potential of providing secure access to information when and where it is needed.

\section{Acknowledgement}

We would like to acknowledge Megan Bell, who assisted us with formulating our search strategy. We also appreciate the guidance and support of the entire Yearbook editorial team, especially Brigitte Séroussi, Lina Soualmia, Adrien Ugon, and Martina Hutter, as well as the reviewers who participated in the selection process of best papers.

\section{References}

1. Bloomrosen M, Detmer D. Advancing the framework: use of health data--a report of a working conference of the American Medical Informatics Association. J Am Med Inform Assoc 2008;15(6):715-22.

2. Safran C, Bloomrosen M, Hammond WE, Labkoff S, Markel-Fox S, Tang PC, et al. Toward a national framework for the secondary use of health data: an American Medical Informatics Association White Paper. J Am Med Inform Assoc 2007;14(1):1-9.

3. Vest JR, Campion TR Jr., Kaushal R. Challenges, alternatives, and paths to sustainability for health information exchange efforts. J Med Syst 2013;37(6):9987.

4. Arzt NH. The new alphabet soup: models of data integration, part 2. J Healthc Inf Manag 2006;20(2):9-11.

5. Arzt NH. The new alphabet soup: models of data integration, part 1. J Healthc Inf Manag 2006;20(1):15-8.

6. El Emam K, Rodgers S, Malin B. Anonymising and sharing individual patient data. BMJ 2015;350:h1139.

7. Gold M, McLaughlin C. Assessing HITECH Implementation and Lessons: 5 Years Later. Milbank Q 2016;94(3):654-87.

8. Calderwood SB, Murray BE, Chambers HF. The $21^{\text {st }}$ Century Cures Act. N Engl J Med 2015;373(17):1679.

9. Bierer BE, Barnes M, Lynch HF. Revised 'Common Rule' shapes protections for research participants. Health Aff (Millwood) 2017;36(5):784-8.

10. Modifications to the HIPAA Privacy, Security, Enforcement, and Breach Notification rules under the Health Information Technology for Economic and Clinical Health Act and the Genetic Information Nondiscrimination Act; other modifications to the HIPAA rules. Federal register 2013;78(17):5565-702.

11. Kloss LL, Brodnik MS, Rinehart-Thompson LA. Access and disclosure of personal health information: A challenging privacy landscape in 2016-2018. Yearb Med Inform 2018:60-6.

12. Ji H, Yoo S, Heo EY, Hwang H, Kim JW. Technology and Policy Challenges in the Adoption and Operation of Health Information Exchange Systems. Healthc Inform Res 2017;23(4):314-21.

13. De Pietro C, Francetic I. E-health in Switzerland: The laborious adoption of the federal law on electronic health records (EHR) and health information exchange (HIE) networks. Health Policy 2018;122(2):69-74.

14. Downing NL, Adler-Milstein J, Palma JP, Lane S, Eisenberg,M, Sharp C, et al. Health information exchange policies of 11 diverse health systems and the associated impact on volume of exchange. J Am Med Inform Assoc 2017;24(1):113-22.

15. HIMA e-HIM Personal Health Record Work Group. Defining the Personal Health Record. J AHIMA 2005;76(6):24-5.

16. Snyder C, Wu AW. Users' Guide to Integrating Patient-Reported Outcomes in Electronic Health Records. Baltimore, MD: Johns Hopkins University; 2017.

17. Bell C, Atkinson KM, Wilson K. Modernizing Immunization Practice Through the Use of Based Platforms. J Med Syst 2017;41(4):57.

18. Miyaji A, Nakasho K, Nishida S. Privacy-Preserving Integration of Medical Data : A Practical
Multiparty Private Set Intersection. J Med Syst 2017;41(3):37.

19. Abdulnabi M, Al-Haiqi A, Kiah MLM, Zaidan AA, Zaidan BB, Hussain M. A distributed framework for health information exchange using smartphone technologies. J Biomed Inform 2017;69:230-50.

20. Roehrs A, da Costa CA, da Rosa Righi R. OmniPHR: A distributed architecture model to integrate personal health records. J Biomed Inform 2017;71:70-81.

21. Mertz L. (Block) Chain Reaction: A Blockchain Revolution Sweeps into Health Care, Offering the Possibility for a Much-Needed Data Solution. IEEE Pulse 2018;9(3):4-7.

22. Park Y, Park Y. A Selective Group Authentication Scheme for IoT-Based Medical Information System. J Med Syst 2017;41(4):48.

23. Mohit P, Amin ., Karati ., Biswas GP, Khan MK. A Standard Mutual Authentication Protocol for Cloud Computing Based Health Care System. J Med Syst 2017;41(4):50.

24. Aslam MU, Derhab A, Saleem K, Abbas H, Orgun M, Iqbal W, et al. A Survey of Authentication Schemes in Telecare Medicine Information Systems. J Med Syst 2017;41(1):14.

25. Lee HJ, Wu Y, Zhang Y, Xu J, Xu H, Roberts $\mathrm{K}$. A hybrid approach to automatic de-identification of psychiatric notes. J Biomed Inform 2017;75S:S19-S27.

26. Klein DM, Pham K, Samy L, Bluth A, Nazi KM, Witry M, et al. The Veteran-Initiated Electronic Care Coordination: A Multisite Initiative to Promote and Evaluate Consumer-Mediated Health Information Exchange. Telemed J E Health 2017;23(4):264-72.

27. Boockvar KS, Ho W, Pruskowski J, DiPalo KE, Wong JJ, Patel J, et al. Effect of health information exchange on recognition of medication discrepancies is interrupted when data charges are introduced: results of a cluster-randomized controlled trial. J Am Med Inform Assoc 2017;24(6):1095101.

28. Hohmeier KC, Spivey CA, Boldin S, Moore TB, Chisholm-Burns M. Implementation of a health information exchange into community pharmacy workflow. J Am Pharm Assoc (2003). 2017;57(5):608-15.

Correspondence to:

Meryl Bloomrosen

E-mail:meryl_bloomrosen@premierinc.com 
Appendix: Content Summaries of Selected Best Papers for the IMIA Yearbook 2018, Section Health Information Management

\author{
Roehrs A, da Costa CA, da Rosa Righi R \\ OmniPHR: A distributed architecture model \\ to integrate personal health records
}

\section{J Biomed Inform $2017 \mathrm{Jul} ; 71: 70-81$}

The authors discuss a distributed architecture model, called OmniPHR, to integrate personal health records (PHRs). The authors' research goal is to answer how to have a single view of a PHR that is up-to-date and interoperable for patients and providers. The proposed model focuses on a distributed approach where patients can maintain a unified view of their health history, from any device anywhere. The approach recognizes current challenges since patients' health data are collected throughout their lives, across the care continuum, and come from multiple and diverse sources, including clinicians, laboratories, clinics or hospitals, and data from sensors that monitor the patients' health. The article summarizes the main concepts, challenges, and models that support the authors' proposal; explains the most significant related work; presents the foundational technologies for model development; details the architecture model; provides the evaluation and methodology of the study; summarizes the results and discusses the impacts, limitations, and future directions; and presents the conclusions of their work.

Setting the stage for their proposal of a computer architecture model for PHRs based on a distributed P2P (peer-to-peer) network system, the authors apply the International Organization for Standardization's (ISO) Technical Committee (TC) 14639 (Health informatics - Capacity-based eHealth architecture roadmap - Part 2: Architectural components and maturity model) definitions for EHRs and PHRs. The authors include discussions about the limitations and the challenges of EHRs and PHRs. A summary of other models described in the literature is also included. The authors discuss the technologies that complete their proposed solution and how they are interconnected with the proposed model. These technologies include: Blockchain, Routing Overlay, openEHR standard, Chord algorithm, and Publish-Subscribe systems. Following the discussion of the model and technologies, the authors provide an additional description of the model's purpose (to allow a unified view of health records which are distributed in several health organizations) and they address the challenges regarding a distributed architecture that is scalable, elastic, and interoperable.

The next section of the paper focuses on the modules and components of OmniPHR design and includes descriptions of each. The authors describe the use of the modeling and profiling methodology to evaluate mobile applications. Their goal is to describe and evaluate scenarios of use where OmniPHR can be applied. The authors also describe and depict the mathematical systems analysis that was undertaken and then provide an extensive discussion of the findings and results. Limitations of the model are described and the authors identify challenges and opportunities. For example, one key challenge for the model is the need to verify the identity and authenticity of the data informants (sources). The need to assure data validity, chain of trust, and security and privacy are also discussed and the need for further testing for security and privacy is noted.

\section{Klein DM, Pham K, Samy L, Bluth A, Nazi KM, Witry M, Klutts JS, Grant KM, Gundlapalli AV, Kochersberger G, Pfeiffer L, Romero S, Vetter B, Turvey CL}

The veteran-initiated electronic care coordination: a multisite initiative to promote and evaluate consumer-mediated health information exchange

\section{Telemed J E Health 2017 Apr ;23(4):264-27}

This pilot study examines the potential of consumer-mediated health information exchange, which gives patients access and control of their health data for promoting continuity of care. Although veterans receive most of their care at the Veterans' Affairs (VA) facilities, many veterans, referred to as 'dual use', receive some care outside the
VA. The VA Office of Rural Health and the Health and Human Services Office of the National Coordinator for Health IT partnered to promote the use of My HealtheVet's Blue Button capability to facilitate transfer of VA health information to non-VA providers to improve care coordination for rural dual-use veterans. The VA launched the Blue Button feature in My HealtheVet, the VA's patient portal, in August 2010. In 2013, a Continuity of Care Document (CCD) in standardized format became available. The VA CCD includes essential information (allergies, medications, diagnoses, immunizations, recent lab results, vital signs, history of procedures, and encounters) from the VA's electronic health record (EHR) that is accessible via the Blue Button.

In this study, VA facilities and rural community healthcare organizations collaborated to develop optimal processes for information exchange. The researchers also engaged and trained veterans in health information sharing (i.e., how to use the Blue Button). The project developed methods for evaluating patient and provider impact of this sharing. The goals of the project were to: (1) train dual-use rural veterans to use the VA's My HealtheVet Blue Button capabilities to promote consumer-mediated HIE of their VA CCD with their non-VA care providers, and (2) evaluate if the availability of VA information at a community clinical encounter impacted the care received.

The authors provided details about how these processes were undertaken and accomplished. Approaches and methods available for veterans to share data with non-VA providers varied and veterans were trained in these processes. Veterans were asked to complete a brief questionnaire after training to evaluate their experiences. Non-VA ("community") providers were also asked to complete a questionnaire to help assess provider satisfaction with the CCD and whether the provider believed the CCD had an impact on the care provided. Detailed analyses were conducted in the following areas: patient characteristics and perceptions of provider communication; patient training evaluation; and data sharing at community non-VA provider visits. Study limitations (such as site variation for patient engagement/training; lack of a comparison 
group; and potential for participant selection bias (veterans' level of interest in health and technology) were described.

The authors conclude that the pilot demonstrated the feasibility and value of patient access to a standard CCD to facilitate information sharing between VA and nonVA providers. With brief training, veterans were able to generate their CCD in My HealtheVet, share it with non-VA providers, and benefit from improved communication about medications and reduced laboratory test duplication. Thus, the authors found that there is patient and provider support for consumer-mediated HIE and they noted that this type of HIE requires outreach and targeted education.

\section{Boockvar KS, Ho W, Pruskowski J, DiPalo KE, Wong JJ, Patel J, Nebeker JR, Kaushal $R$, Hung W}

\section{Effect of health information exchange on recognition of medication discrepancies is interrupted when data charges are in- troduced: results of a cluster-randomized controlled trial}

\section{J Am Med Inform Assoc 2017 Nov 1;24(6):1095-101}

The authors explored the effect of health information exchange (HIE) on medication prescribing for hospital inpatients in a Veterans Administration hospital in a cluster-randomized controlled trial and examined the prescribing effect of availability of information from a large pharmacy insurance plan in a natural experiment. They recognized that a key step in medication reconciliation is information-gathering from various sources such as patients, family members, providers' offices, health care facilities, pharmacies, and prescription coverage plans and postulated that [regional] HIEs could improve medication safety by facilitating reconciliation of medication information from multiple sources at the time of patient care. The researchers hypothesized that HIE would raise the impact of medication reconciliation for hospitalized veterans who utilize VA and non-VA services on discrepancies between preadmission and inpatient medication regimens (primary outcome) and reduction of ADEs (secondary outcome). Patients were assigned to intervention or control groups according to the hospital unit(s) to which they were admitted.

The study describes the methodology, protocols, and quality controls in detail. For patients assigned to the intervention group (HIE-enhanced medication reconciliation), an intervention pharmacist conducted HIE-enhanced medication reconciliation, following a structured protocol. For patients assigned to usual care, the intervention pharmacist performed the structured medication reconciliation protocol but without access to the information available from HIE. The study defined medication discrepancies as differences between a patient's prehospital medication list and the medications received in the hospital. The discrepancies were initially identified and recorded by the unblinded intervention pharmacist at the time of admission medication reconciliation. The unit of observation was hospitalization episode. For each study group, descriptive statistics were used to describe patient and hospitalization characteristics, time from hospital admission to medication reconciliation, and house staff rectification of medication discrepancies.

Results indicated that there were no significant differences between intervention and control groups in baseline characteristics. The mean time from hospital admission to medication reconciliation in both intervention and control groups was the same. The researchers also found that there were no differences between intervention and control groups in numbers of verbal or co-signature alerts that the intervention pharmacist provided to physicians. However, patients who received HIE-enhanced medication reconciliation with pharmacy insurance data available had greater risk-weighted medication discrepancies identified than those who received usual care. There were no differences in ADEs between those assigned to HIE-enhanced medication reconciliation and those assigned to usual care, or between those who received HIE-enhanced medication reconciliation with pharmacy insurance plan data available and those who received usual care.

Study limitations were described and include: low house staff responsiveness to medication discrepancy information; de- layed mean time from hospital admission to the intervention pharmacist's medication reconciliation; and low level of medication information in the HIE. The authors noted a strength of their study was that they tested the effect of HIE in potentially high-impact circumstances (medication prescribing at the time of hospital admission) and did not depend on voluntary HIE access by the user (the intervention pharmacist was obligated to access HIE for all intervention patients). The authors conclude that HIE may improve outcomes of medication reconciliation. However, the authors raise concerns related to potentially harmful consequences of charging for access to information (in this case payment data) and related to information blocking practices.

Downing NL, Adler-Milstein J, Palma JP, Lane S, Eisenberg M, Sharp C; Northern California HIE Collaborative, Longhurst CA Health information exchange policies of 11 diverse health systems and the associated impact on volume of exchange

\section{J Am Med Inform Assoc 2017 Jan;24(1):113-22}

Focusing on health information exchange (HIE) across 11 health systems that all used the same electronic health record, the authors conducted a retrospective time series analysis of the effect on the monthly volume of clinical summaries exchanged of automatic querying and different processes for patient consent. The consent processes included using the general consent for treatment to cover the consent for HIE vs. requesting specific consent for each individual need for HIE. The researchers did not assess degree of use or usefulness of the information exchanged (care summaries), organizational decision-making processes, or generalizability to other vendors.

Given the policy levers and financial incentives available to providers, a variety of approaches to health information exchange (including community-based exchange networks, enterprise-based exchange networks, and electronic health record (EHR) vendor-based platforms) have been implemented. While each approach reflects various technological solutions, there are 
also operational, logistical, and management processes, and decisions that are embedded within each exchange. The study objective was to examine the relationship between electronic exchanges of patient health information across organizations and organizational HIE policy decisions.

The researchers looked at data on organization-level HIE policy decisions and their impact on HIE volume from a diverse set of health care systems using the same EHRbased HIE platform. The focus of the policies was on whether to automatically search for information from other organizations whenever a patient with data in those organizations presented for care, and whether to require HIE-specific patient consent. Their research questions were: 1) What proportion of organizations chose to engage in auto- matic querying and what is the associated impact on the volume of clinical summary exchange? 2) When automatic querying is enabled, what proportion of patient linkages are established automatically (representing information at another institution that the provider did not know to seek) vs. manually requesting the information (representing information the provider knew to seek)? and (3) What proportion of organizations chose not to require specific patient consent for HIE and what is the associated impact on the volume of clinical summary exchange?

The study covered a 2-year period from January 1, 2013, through February 28, 2015, and included linkages made and clinical summaries transferred across all clinical settings within each institution (such as outpatient clinics or other settings, emergency departments, and inpatient stays). Study limitations included: the inability to normalize exchange volume to account for the volume of patient care; inability to determine the extent to which clinical summaries were used for patient care; lack of information on how providers decided to implement their approach (auto-query or consent); and inclusion of only institutions using a single vendor-based HIE platform.

The authors found that automatic querying and not requiring specific consent for HIE for each individual care episode appeared to substantially increase exchange volume. They conclude that these organizational HIE policy decisions impact the volume of exchange, and ultimately the information available to providers to support optimal care. 